\title{
Labyrinthine patterns transitions
}

\author{
S. Echeverría-Alar $\odot$ and M. G. Clerc $\odot$ \\ Departamento de Física and Millenium Institute for Research in Optics, Facultad de Ciencias Físicas y Matemáticas, \\ Universidad de Chile, Casilla 487-3, Santiago, Chile
}

(Received 30 August 2020; accepted 23 November 2020; published 7 December 2020)

\begin{abstract}
Macroscopic systems with injection and dissipation of energy exhibit intricate dissipative structures. Labyrinthine patterns are disordered spatial structures arising into homogeneous media that show a short-range order. Here, we investigate the stability properties, classification, and transitions of labyrinthine patterns. Based on a prototype pattern forming model, we characterize the existence of three types of labyrinthine patterns-fingerprint type, glassy, and scurfy-and reveal the bifurcation diagram. The defects density, free energy, structure factor, and correlation length are used as order parameters.
\end{abstract}

DOI: 10.1103/PhysRevResearch.2.042036

Nature is full of patterns such as dunes, animal fur, fish skin, snowflakes, pigmentation on mollusk shells, fir waves, mountain ripples, and clouds, which have attracted attention due to their regularities or irregularities that generate the diversity of forms [1-5]. All these physical systems correspond to macroscopic systems with injection and dissipation of energy. Out-of-equilibrium systems are characterized in general by exhibiting pattern formation as a result of spontaneous spatial breaking symmetry of a homogeneous state [1-5]. Near this transition, the observed equilibrium patterns are generally striped, hexagonal, square, and superlattice. A unified strategy to describe the emergence and dynamics of these simple patterns is the amplitude equations of critical modes [1-5]. By increasing energy injection, pattern forming systems exhibit more complex patterns, characterized by a large number of defects. Indeed, far from the primary spatial instabilities, disordered patterns arise into homogeneous media. An example of these types of complex patterns is the so-called labyrinthine patterns. Figure 1 shows examples of labyrinthine patterns observed in nature. These patterns have been observed in mussel beds [6], cardiovascular calcification [7], phytomass [8], microemulsions [9], fish skin [10], fluid convection [11], Langmuir monolayer [12], magnetic fluids [13], chemical reactions [14], and cholesteric liquid crystals [15], to mention a few. Hence, labyrinthine patterns are a robust phenomenon of nature. Intuitively, in all the aforementioned systems, the observed patterns are denominated labyrinths. However, a precise definition is not available. A proposition of a labyrinthine pattern is a spatial state that shows a short-range order and a powdered spatial spectrum [16]. Notwithstanding the above, when one considers a hexagonal structure with defects, it satisfies the previous definition and is not an intuitive labyrinth

Published by the American Physical Society under the terms of the Creative Commons Attribution 4.0 International license. Further distribution of this work must maintain attribution to the author(s) and the published article's title, journal citation, and DOI. structure. The previous definition can be amended by specifying that a single wave number characterizes short-range order. The characterization of different types of labyrinth patterns, their properties as a state of equilibrium, and transitions between them has not been established.

The purpose of this Rapid Communication is to describe different types of labyrinthine patterns and characterize the transitions between them. Based on a prototype pattern forming model, the Turing-Swift-Hohenberg equation, we characterize the existence of three types of labyrinthine patterns (fingerprint-type, glassy, and scurfy) and reveal their transitions. The defects density, free energy, structure factor, and correlation length are used as order parameters. Fingerprint-type labyrinths arise as a result of the emergence of bound states between defects. This is a supercritical transition, which precedes freezing of the coarsening process that characterizes striped patterns [17]. When increasing the bifurcation parameter, the transition between the fingerprinttype and glassy patterns is of the second-order type, which is detected by means of the correlation length. The structure factor allows us to characterize the transition between glassy and scurfy labyrinthine patterns. Free energy allows us to envisage the complex organization of the different labyrinthine patterns.

Let us consider a prototype model of pattern formation, the dimensionless Turing-Swift-Hohenberg equation [18]. This model equation accounts for the dynamics of a real order parameter deduced originally to describe the pattern formation on Rayleigh-Bénard convection [18,19], which reads

$$
\partial_{t} u=\epsilon u-u^{3}-v \nabla^{2} u-\nabla^{4} u,
$$

with $u=u(x, y, t)$ a real scalar field, and $x, y$, and $t$ are spatial coordinates, and time, respectively. $\epsilon$ is the bifurcation parameter. The parameter $v$ stands for the diffusion coefficient $(v<0)$; when this parameter is positive $(v>0)$, it induces an antidiffusion process. Notice that the above model is the simplest isotropic, reflection symmetry, and nonlinear equation that presents patterns and localized states [2]. In the last decade, it has been established that Eq. (1) had already been 


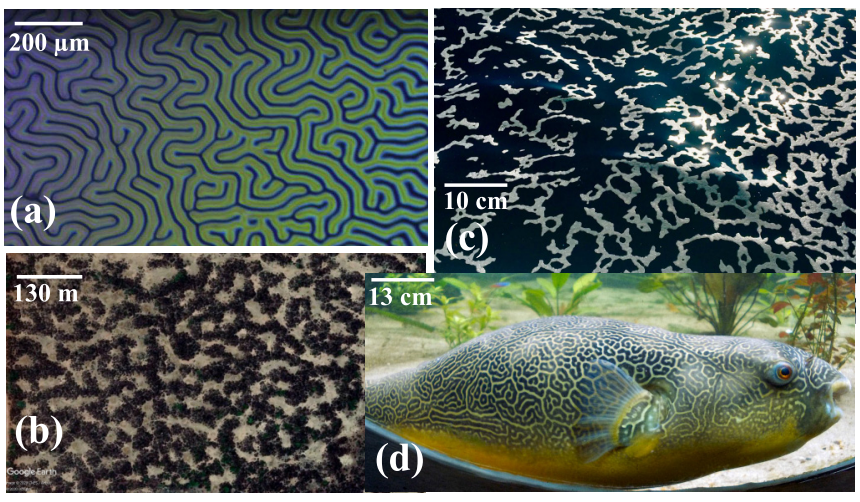

FIG. 1. Labyrinthine patterns. (a) Chiral liquid crystal labyrinthine pattern (courtesy of Gregorio Gonzalez). (b) Vegetation patterns observed in Niger using Google Earth (coordinates $12^{\circ} 27^{\prime} 50.61^{\prime \prime} N 3^{\circ} 18^{\prime} 30.76^{\prime \prime} \mathrm{E}$ ). (c) Seafoam patterns (courtesy of Randall Straka). (d) Giant puffer fish (courtesy of Chiswick Chap).

constructed by Turing, but was unpublished [19]. Note that Eq. (1) was published 23 years after Turing's death [18]. Observe that model equation (1) is of variational nature, that is, $\partial_{t} u=-\delta \mathcal{F} / \delta u$ where $\mathcal{F}$ is the Lyapunov function

$$
\mathcal{F}[u]=\iint\left(-\epsilon \frac{u^{2}}{2}+\frac{u^{4}}{4}-v \frac{(\nabla u)^{2}}{2}+\frac{\left(\nabla^{2} u\right)^{2}}{2}\right) d x d y .
$$

Hence, the dynamics of model equation (1) is characterized by the minimization of the free energy $\mathcal{F}$.

A trivial solution of model equation (1) is the zero solution, $u(x, y, t)=0$. This solution is stable for $\epsilon<\epsilon_{c} \equiv$ $-v^{2} / 4$. For $\epsilon=\epsilon_{c}$, the homogeneous solution becomes unstable and gives rise to the emergence of a striped pattern with wavelength $\left|\vec{k}_{c}\right|=\sqrt{v / 2}[2,3]$. This spatial supercritical instability is characterized by the emergence of domains of striped patterns with different orientations and the same wavelength. These domains are randomly distributed. Defects that reconcile different pattern orientations separate the domains. These are local disclinations and extended grain boundaries; both of amplitude and phase. Also, dislocations appear that fix local discrepancies in wavelength (see textbook [3], and references therein). The interaction of these defects causes domains to grow over time. This process is self-similar, usually called coarsening process, which is characterized by the grain-boundaries perimeter growing as a power law of time $t^{1 / 3}$ [17]. Note that phase separation in supersaturated solids $[20,21]$ and the formation of localized patterns in optics $[22,23]$ present a coarsening process, which is governed by the same critical exponent. Hence, in a finite-size system, the final equilibrium reached is a striped pattern (monodomain). Figure 2(a) shows the typical observed striped pattern. The orientation of these patterns only depends on the initial condition. The modulus Fourier transform of this type of pattern is characterized by exhibiting two maximums [see the upper inset panel in Fig. 2(a)]. In order to describe the short-range order of the pattern, we introduce the averaged windowed Fourier transform by considering a large number of boxes of the same length $l$, calculating the Fourier transform, rotating the wave-vector space so that a peak of the transformation is
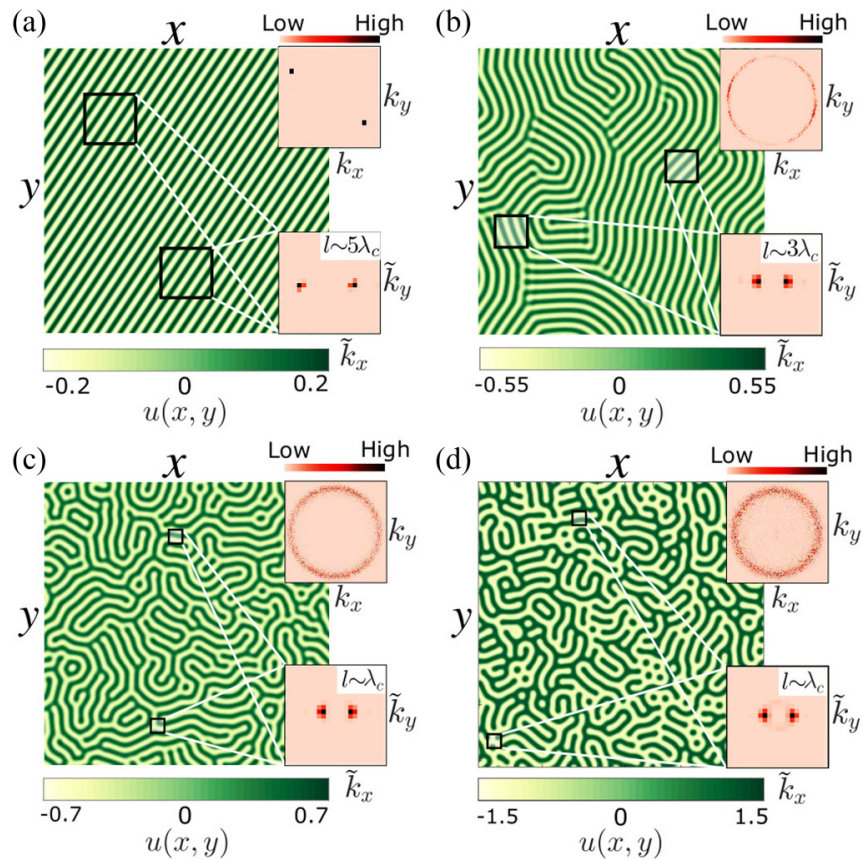

FIG. 2. Equilibrium patterns of the dimensionless Turing-SwiftHohenberg equation (1) with $v=1$. Surface plots of (a) striped pattern $\epsilon=-0.22$, (b) fingerprint-type labyrinthine pattern $\epsilon=$ -0.16 , (c) glassy labyrinthine pattern $\epsilon=0.02$, and (d) scurfy labyrinthine pattern $\epsilon=1.15$. The upper and lower insets account for the modulus of global and averaged windowed Fourier transforms, respectively. $\lambda_{c}$ and $l$ are the critical wavelength $\left(2 \pi /\left|\vec{k}_{c}\right|\right)$ and the size of the window in the averaged windowed Fourier transform, respectively.

always horizontal, and averaging over these transforms. The lower panel of Fig. 2(a) illustrates this transform.

Unexpectedly, when the bifurcation parameter $\epsilon$ is increased, the interaction of defects freezes [24], that is, the defects present bound states. In this region of parameters, starting with the zero solution perturbed with a small initial noise, the system exhibits labyrinth solutions as equilibria. Figure 2 shows the different labyrinthine patterns observed in the Turing-Swift-Hohenberg equation. By using the global and averaged windowed Fourier transform, we can verify that the observed patterns are disordered and show a short-range order characterized by a single wave number. All numerical simulations were implemented using a pseudospectral code with the Runge-Kutta order-4 algorithm. In the present study, $u=0$ with a small random disturbance is always considered as the initial condition.

To verify that the labyrinthine patterns are equilibrium states, we have conducted two analyses. First, monitoring the evolution of free energy over time $\mathcal{F}[u(x, y, t)]$, which should have the tendency to decrease and settle at an asymptotic value. Indeed, the free energy decreases as the defects are annihilated or reach their final position. Figure 3 shows the evolution $\mathcal{F}[u]$ as a function of time. And second, by calculating the linear stability spectrum of the labyrinth solution (cf. upper inset in Fig. 3). Both analyses allow us to conclude that labyrinthine patterns are stable. 


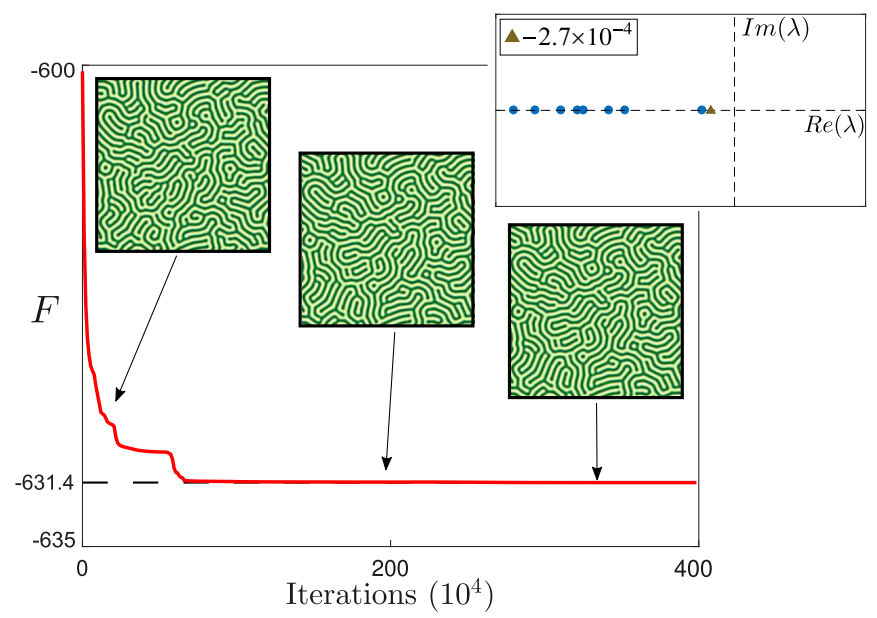

FIG. 3. Temporal evolution of the free energy $\mathcal{F}[u(x, y, t)]$ for a labyrinth state of the Turing-Swift-Hohenberg equation (1) with $v=$ 1 and $\epsilon=0.02$. The upper inset corresponds to the linear stability spectrum of the labyrinth solution for large times. The lower insets show the labyrinthine pattern for three different instances.

When defects begin to freeze, $\epsilon=\epsilon_{\mathrm{SFP}}$, the system features large domains of striped patterns mainly separated by amplitude grain boundaries. We have called these structures fingerprint-like labyrinthine patterns, due to their similarity to fingerprints. Figure 2(b) shows a fingerprint-type pattern and their respective Fourier transforms. To characterize the emergence of these patterns, we have measured the defects density $f_{d}$, based on the orientational field of the pattern [25], as a function of the bifurcation parameter. Figure 4(a) summarizes $f_{d}$ as a function of the bifurcation parameter. Observe that the transition between fingerprint-type and striped patterns is of a supercritical type and described by a critical exponent $1 / 2$, as shown by the continuous curve [cf. Fig. 4(a)]. Notice that at this critical point, the striped patterns do not become unstable, and even for higher bifurcation parameters, they coexist with labyrinthine patterns. Likewise, if one calculates the correlation length $\xi$ [26] as a function of the bifurcation parameter [see Fig. 4(a)], we observe that this length diverges in the transition between fingerprint-type and striped patterns with a $-1 / 2$ power law. When increasing the bifurcation parameter, we observe that the rate of the defects density decreases; particularly from $\epsilon>\epsilon_{\mathrm{FPG}}$, no grain boundaries were observed. Then, the patterns in this region are characterized by presenting a large number of local defects. We have termed this type of pattern as glassy labyrinths, due to its amorphous structure. Figure 2(c) shows a typical glassy labyrinthine pattern. The correlation length close to $\epsilon_{\mathrm{FPG}}$ exhibits a slight local maximum [cf. inset in Fig. 4(a)], which is a peculiarity of glassy-type second-order transitions [27]. To analyze this transition more carefully, we have monitored the time evolution of the correlation length. Figure 4(b) shows the evolution of the correlation length as a function of the bifurcation parameter. From this chart, we infer that the correlation length variations are almost negligible for glassy labyrinthine patterns. Indeed, the glassy state corresponds practically to an instantly frozen state. In brief, the fingerprint-type and glassy labyrinthine patterns can be distinguished by the prevalence
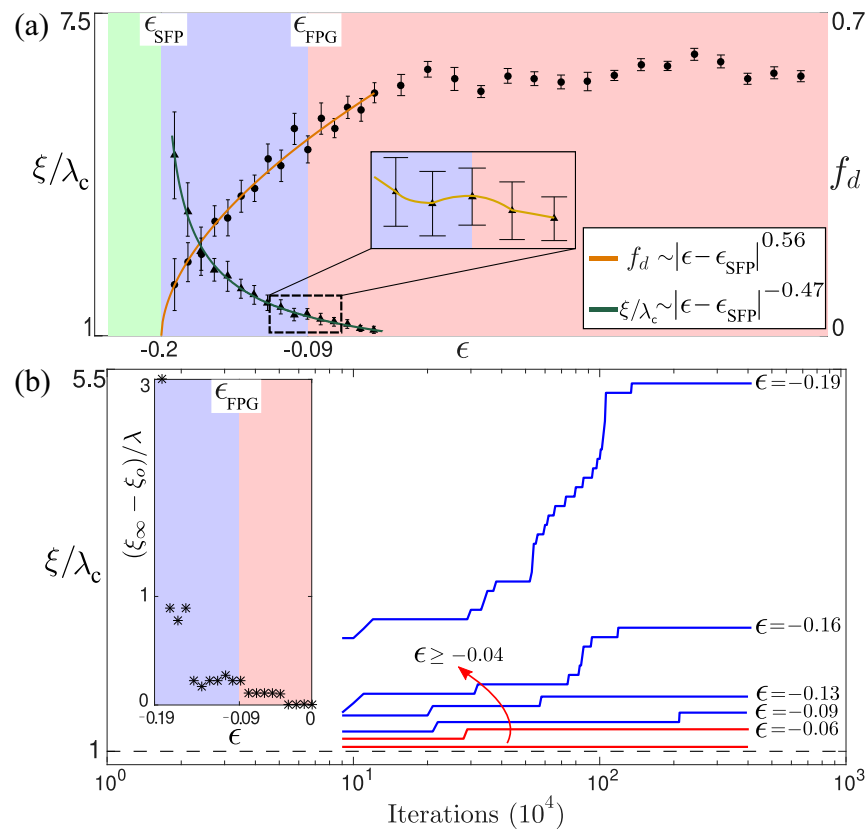

FIG. 4. Bifurcation diagram of labyrinthine patterns. (a) The correlation length $\xi$ and defects density $f_{d}$ as a function of the bifurcation parameter $\epsilon$. The circles $(\bullet)$ and triangles $(\triangle)$ account for the correlation length and defects density, respectively. The error bars account for the standard deviation obtained after analyzing 50 numerical realizations. $\epsilon_{\mathrm{SFP}}$ and $\epsilon_{\mathrm{FPG}}$ account for the transition points between striped and fingerprint-type labyrinthine patterns, and fingerprint-type and glassy labyrinthine patterns, respectively. The solid curves describe the fitting for the defects density and correlation length. The inset accounts for the magnification of the correlation length close to the transition from fingerprint-type to glassy patterns. (b) Temporal evolution of the correlation length $\xi$ as a function of the bifurcation parameter $\epsilon$. The inset shows the initial $\xi_{0}$ and equilibrium $\xi_{\infty}$ correlation length difference as a function of the bifurcation parameter.

of amplitude grain boundaries and the relaxation dynamics to their respective equilibria.

By further increasing the bifurcation parameter, we observe that the glassy patterns maintain the same structure. However, from a critical value, $\epsilon>\epsilon_{G S}$, we begin to find the appearance of circular spots embedded in the labyrinth pattern. We have called this type of structure scurfy labyrinthine patterns. Figure 2(c) shows a typical scurfy labyrinthine pattern. As a result of the presence of spots we are not able to compute the defects density and correlation length. To analyze the emergence of circular spots onto labyrinthine patterns, let us consider the structure factor

$$
S(k)=\int_{-\pi}^{\pi}\left|\frac{1}{\sqrt{\pi}} \int u(x, y, t) e^{i \vec{k} \vec{r}} d x d y\right| k d \theta,
$$

where $k=|\vec{k}|$ and $\theta$ are the polar coordinate representation of wave-vector space $\vec{k}$. The typical structure factors $S(k)$ for the different labyrinthine patterns are shown in the insets of Fig. 5. Note that for fingerprint-type and glassy patterns only one dominant peak is observed, while for scurfy labyrinthine patterns there are two peaks. This second peak close to $k=0$ is a consequence of the effect of circular spots and 


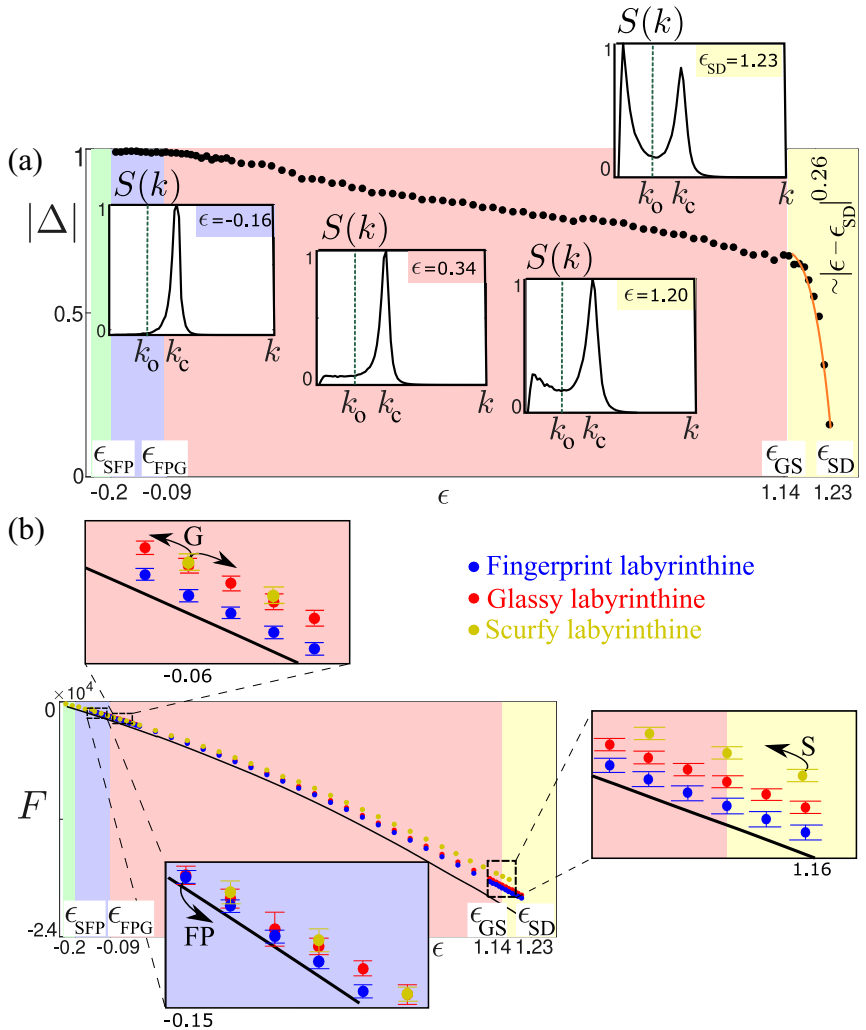

FIG. 5. Characterization of labyrinthine patterns. (a) The normalized area difference between the peaks, $\Delta(\epsilon) \equiv\left(\int_{k_{o}}^{\infty} S d k-\right.$ $\left.\int_{0}^{k_{o}} S d k\right) / \int_{0}^{\infty} S d k$ as a function of the bifurcation parameter. The insets correspond to normalized structure factors at different values of the bifurcation parameter. The continuous curve $\Delta(\epsilon) \approx$ $\left|\epsilon-\epsilon_{S D}\right|^{0.26}$ accounts for the fitting obtained for the area difference near the disappearance of the scurfy patterns. $\epsilon_{G S}$ and $\epsilon_{S D}$ stand for the transition points between glassy and scurfy labyrinths, and scurfy labyrinths and their disappearance, respectively. (b) Free energy $\mathcal{F}$ of the different equilibria as a function of the bifurcation parameter $\epsilon$. The solid curve accounts for the energy of the striped pattern. The circles account for the different labyrinthine patterns ( $\circ$ fingerprint-type, $\circ$ glassy, and $\circ$ scurfy). The error bars show the standard deviation obtained from 30 realizations. Insets stand for the magnification of the chart in the respective regions.

localized structures. Introducing the normalized area difference between the peaks,

$$
\Delta(\epsilon) \equiv \frac{\int_{k_{o}}^{\infty} S(k) d k-\int_{0}^{k_{o}} S(k) d k}{\int_{0}^{\infty} S(k) d k},
$$

one can monitor the emergence of the second peak, where $k_{o}$ is an intermediate wave number between peaks. Figure 5 shows the evolution of the area difference $\Delta$ as a function of $\epsilon$. This indicator presents a sharp decrease at $\epsilon=\epsilon_{G S}$. For $\epsilon_{G S}<\epsilon<\epsilon_{S D}$, we observe scurfy labyrinthine patterns. In this region of parameters, we observe that the labyrinthine patterns coexist with the uniform state and localized structures $[28,29]$. Hence, $\epsilon_{G S}$ accounts for the transition between glassy and scurfy labyrinthine patterns. When $\epsilon>\epsilon_{S D}$, the scurfy labyrinthine patterns become unstable by shrinking, and forming a gas of localized spots. The inverse process, that is, how a labyrinthine pattern is generated deterministically from a localized structure, is well known as invagination [29]. Close to the disappearance point of the labyrinthine patterns, we find $\Delta(\epsilon)$ satisfies a power law $1 / 4$ [see Fig. 5(a)].

The Turing-Swift-Hohenberg model equation (1) is variational. Then, we can use the free energy $\mathcal{F}$ to characterize different equilibria. Figure 5(b) illustrates the free energy $\mathcal{F}$ of the different equilibria as a function of the bifurcation parameter $\epsilon$. From this chart, we conclude that the striped pattern is the most stable state. The fingerprint-type labyrinthine patterns are the most stable labyrinthine state. Note that close to $\epsilon_{\mathrm{FPG}}$, the energy of fingerprint-type labyrinths merges with the energy of glassy and scurfy ones. The energy of the glassy labyrinths is always less than the energy of the scurfy labyrinths. Therefore, considering different initial conditions, the system presents a large number of equilibria with varying levels of energy.

In conclusion, out-of-equilibrium systems exhibit complicated disordered patterns. One of the patterns observed in various physical contexts is the so-called labyrinth pattern. We establish these labyrinthine patterns as a spatial state that shows a short-range order characterized by a single wave number and a powdered spectrum. The consideration of a local single wave-number behavior allows the distinction between labyrinths and disordered hexagonal patterns, the latter characterized locally by three wave numbers. Based on a prototype pattern forming model, the Turing-Swift-Hohenberg equation, we investigate the stability properties, classification, and transitions between labyrinthine patterns. The observation of three types of labyrinthine patterns - fingerprint-type, glassy, and scurfy - are established. The density of defects, free energy, structure factor, and correlation length allow us to reveal the bifurcation diagram. The mentioned order parameters, except free energy, together with techniques of global and averaged windowed Fourier transforms can also be used to characterize stationary labyrinths and their transitions in nonvariational systems $[16,30]$.

The authors thank Gregorio Gonzalez for fruitful discussions. The authors acknowledge the financial support of FONDECYT project 1180903 and Millennium Institute for Research in Optics (MIRO). S.E.-A. acknowledges the financial support of ANID by Beca Doctorado Nacional 202021201376.
[1] G. Nicolis and I. Prigogine, Self-Organization in NonEquilibrium Systems (Wiley, New York, 1977).

[2] M. Cross and H. Greenside, Pattern Formation and Dynamics in Non-Equilibrium Systems (Cambridge University Press, New York, 2009).
[3] L. Pismen, Patterns and Interfaces in Dissipative Dynamics (Springer, Berlin, 2006).

[4] M. I. Rabinovich, A. B. Ezersky, and P. D. Weidman, The Dynamics of Patterns (World Scientific, Singapore, 2000). 
[5] D. Walgraef, Spatio-Temporal Pattern Formation: With Examples from Physics, Chemistry, and Materials Science (Springer Science \& Business Media, Berlin, 2012).

[6] Q. X. Liu, P. M. Herman, W. M. Mooij, J. Huisman, M. Scheffer, H. Olff, and J. Van De Koppel, Pattern formation at multiple spatial scales drives the resilience of mussel bed ecosystems, Nat. Commun. 5, 5234 (2014).

[7] A. Yochelis, Y. Tintut, L. L. Demer, and A. Garfinkel, The formation of labyrinths, spots and stripe patterns in a biochemical approach to cardiovascular calcification, New J. Phys. 10, 055022 (2008).

[8] J. von Hardenberg, E. Meron, M. Shachak, and Y. Zarmi, Diversity of Vegetation Patterns and Desertification, Phys. Rev. Lett. 87, 198101 (2001).

[9] I. R. Epstein and V. K. Vanag, Complex patterns in reactive microemulsions: Self-organized nanostructures? Chaos 15, 047510 (2005).

[10] R. Barrio, C. Varea, J. Aragón, and P. Maini, A two-dimensional numerical study of spatial pattern formation in interacting Turing systems, Bull. Math. Biol. 61, 483 (1999).

[11] S. W. Morris, E. Bodenschatz, D. S. Cannell, and G. Ahlers, Spiral Defect Chaos in Large Aspect Ratio Rayleigh-Bénard Convection, Phys. Rev. Lett. 71, 2026 (1993).

[12] Z. Khattari and T. M. Fischer, Shapes of Langmuir monolayer domains in confined geometries, J. Phys. Chem. B 106, 1677 (2002).

[13] R. E. Rosensweig, M. Zahn, and R. Shumovich, Labyrinthine instability in magnetic and dielectric fluids, J. Magn. Magn. Mater. 39, 127 (1983).

[14] K. J. Lee, W. McCormick, Q. Ouyang, and H. L. Swinney, Pattern formation by interacting chemical fronts, Science 261, 192 (1993).

[15] P. Oswald, J. Baudry, and S. Pirkl, Static and dynamic properties of cholesteric fingers in electric field, Phys. Rep. 33767 (2000).

[16] M. Le Berre, E. Ressayre, A. Tallet, Y. Pomeau, and L. Di Menza, Example of a chaotic crystal: The labyrinth, Phys. Rev. E 66, 026203 (2002).
[17] D. Boyer and J. Viñals, Domain coarsening of stripe patterns close to onset, Phys. Rev. E 64, 050101(R) (2001).

[18] J. Swift and P. Hohenberg, Hydrodynamic fluctuations at the convective instability, Phys. Rev. A 15, 319 (1977).

[19] J. H. Dawes, After 1952: The later development of Alan Turing's ideas on the mathematics of pattern formation, Hist. Math. 43, 49 (2016).

[20] M. Lifshitz and V. Slyozov, The kinetics of precipitation from supersaturated solid solutions, J. Phys. Chem. Solids 19, 35 (1961).

[21] C. Wagner, Theorie der Alterung von Niderschlagen durch Umlösen (Ostwald Reifung), Z. Electrochem. 65, 581 (1961).

[22] M. Tlidi, P. Mandel, and R. Lefever, Kinetics of Localized Pattern Formation in Optical Systems, Phys. Rev. Lett. 81, 979 (1998).

[23] M. Tlidi, P. Mandel, M. Le Berre, E. Ressayre, A. Tallet, and L. Di Menza, Phase-separation dynamics of circular domain walls in the degenerate optical parametric oscillator, Opt. Lett. 25, 487 (2000).

[24] D. Boyer and J. Viñals, Grain boundary pinning and glassy dynamics in stripe phases, Phys. Rev. E 65, 046119 (2002).

[25] D. A. Egolf, I. V. Melnikov, and E. Bodenschatz, Importance of Local Pattern Properties in Spiral Defect Chaos, Phys. Rev. Lett. 80, 3228 (1998).

[26] M. C. Cross and D. I. Meiron, Domain Coarsening in Systems Far from Equilibrium, Phys. Rev. Lett. 75, 11 (1995).

[27] E. Donth, The glass transition: Relaxation dynamics in liquids and disordered materials (Springer Science \& Business Media, Berlin, 2013), Vol. 48.

[28] K. Ouchi and H. Fujisaka, Phase ordering kinetics in the SwiftHohenberg Equation, Phys. Rev. E 54, 3895 (1996).

[29] I. Bordeu, M. Clerc, R. Lefever, and M. Tlidi, From localized spots to the formation of invaginated labyrinthine structures in a Swift-Hohenberg model, Commun. Nonlinear Sci. Numer. Simul. 29, 482 (2015).

[30] M. Tlidi, R. Lefever, and A. Vladimirov, On vegetation clustering, localized bare soil spots and fairy circles, Lect. Notes Phys. 751, 381 (2008). 